\title{
COMPLETE MULTIPLIERS
}

\author{
BY
}

\section{J.S. BYRNES}

ABSTRACT. We investigate whether the completeness of a complete orthonormal sequence for $L^{2}(-\pi, \pi)$ is preserved if the sequence is perturbed by multiplying a portion of it by a fixed function. For the particular sequence $\left\{(2 \pi)^{-1 / 2} e^{i n x}\right\}$ we show that given any $\psi \in L^{\infty}(-\pi, \pi)$, except $\psi=0$ a.e., there is a nontrivial portion of $\left\{(2 \pi)^{-1 / 2} e^{i n x}\right\}$ which will maintain completeness under this perturbation.

We investigate whether the completeness of a complete orthonormal sequence (CONS) for $L^{2}(-\pi, \pi)$ (complex-valued) is preserved if the sequence is perturbed by multiplying a portion of it by a fixed function.

To be more precise, given a function $\psi \in L^{\infty}(-\pi, \pi)$ and a $\operatorname{CONS} \Phi=\left\{\phi_{n}\right\}_{-\infty}^{\infty}$ for $L^{2}$ we call a subset $S$ of the set $Z$ of integers a $\Sigma_{\psi, \Phi}$-set (for $L^{2}$ ) if the sequence $\left\{\theta_{n}\right\}$, defined by $\theta_{n}=\psi \phi_{n}$ for $n \in S$ and $\theta_{n}=\phi_{n}$ for $n \notin S$, is also complete in $L^{2}$. If all subsets of $Z$ are $\Sigma_{\psi, \Phi}$-sets we call the pair $(\psi, \Phi)$ a complete pair (for $L^{2}$ ), and if $(\psi, \Phi)$ is a complete pair for all CONS $\Phi$ we call $\psi$ a complete multiplier (for $L^{2}$ ).

The most trivial example of a complete multiplier is the function which is 1 almost everywhere (a.e.), whereas if $\psi$ is 0 a.e. then the empty set is the only $\Sigma_{\psi, \Phi}$-set. Also, if $\psi$ is 0 on a set of positive measure then certainly $(\psi, \Phi)$ cannot be a complete pair for any CONS $\Phi$, since $Z$ itself will not be a $\Sigma_{\psi, \Phi}$-set. However, even for such a $\psi$, and even if the measure of the zero set of $\psi$ is arbitrarily close to $2 \pi$, there will exist nonempty subsets of $Z$ which are $\Sigma_{\psi, \Phi}$ sets.

In [1] it was shown that for $\psi$ to be a complete multiplier it is necessary and sufficient that there exist a complex constant $\alpha$ such that $\operatorname{Re} \alpha \psi \geq 0$ a.e. and either $\operatorname{Im} \alpha \psi>0$ a.e. or $\operatorname{Im} \alpha \psi<0$ a.e. on the zero set of $\operatorname{Re} \alpha \psi$.

We now consider in more detail the case when $\psi$ (which we assume is not the function which is 0 a.e.) is not a complete multiplier. In particular we ask if there always must exist a nontrivial $\Sigma_{\psi, \Phi}$-set (where $S$ is trivial if $S$ is empty or $S=Z$ ).

As a partial answer to this question we first determine when a finite $\Sigma_{\psi, \Phi}$-set

Received by the editors February 9, 1971 and, in revised form, April 29, 1971.

AMS (MOS) subject classifications (1969). Primary 421 7; Secondary 3067.

Key words and phrases. Completeness in $L^{2}$, perturbation, complete orthonormal sequence, Fourier series, perturbation of Fourier series. 
exists and then concentrate our attention on the standard CONS, $\delta=\left\{(2 \pi)^{-1 / 2} e^{i n x}\right\}$. For this special case we are able to prove the result we are seeking, namely:

Main Theorem. There exists a nontrivial $\Sigma_{\psi, \delta}$-set.

As mentioned above, however, before proving this we must investigate the existence of finite $\Sigma_{\psi_{\mathbf{\Phi}} \Phi}$-sets. In this regard we have

Theorem 1. Let $N$ be a positive integer. There is no $1,2,3, \cdots, N$ point $\Sigma_{\psi, \Phi}$-set if and only if for each m-tuple $\left(n_{1}, n_{2}, \cdots, n_{m}\right)$ of (not necessarily distinct) integers, and for eacb $m, 1 \leq m \leq N$, at least one of the following integrals is zero: $\int \bar{\psi} \phi_{n_{i}} \bar{\phi}_{n_{i+1}}, 1 \leq i \leq m$ (where $n_{m+1}=n_{1}, \bar{\psi}$ denotes the complex conjugate of $\psi$, and the range of integration is $(-\pi, \pi)$ ).

Proof. The "if" half of the theorem is not needed in the sequel, so we present here just the proof of the "only if" half. For the interested reader, the remainder of the proof is presented in the Appendix.

The proof will be by induction on $N$, so first suppose that $N=1$. If there is no 1 point $\Sigma_{\psi, \Phi}$-set then for each $n$ there is an $f_{n} \in L^{2}, f_{n}$ not the 0 (a.e.) function, such that

(i) $\int f_{n} \bar{\phi}_{m}=0, m \neq n$, and

(ii) $\int f_{n} \overline{\psi \phi_{n}}=0$.

But (i) says that $f_{n}=c_{n} \phi_{n}$ where $c_{n}$ is a nonzero constant, and then (ii) says $\int \psi\left|\phi_{n}\right|^{2}=0$.

We now assume that the theorem is true for $N=1,2, \cdots, K-1(K \geq 2)$ and prove it for $N=K$. Thus, suppose that there is no $1,2, \cdots, K$ point $\Sigma_{\psi, \Phi}$-set. By the inductive hypothesis we know that the conclusion of the theorem holds for each $m$-tuple of integers, where $1 \leq m \leq K-1$. If we let $\left(n_{1}, n_{2}, \cdots, n_{K}\right)$ be an arbitrary $K$-tuple of integers we know, since there is no $K$ point $\Sigma_{\psi, \Phi^{-s e t}}$, that there is an $f_{K} \in L^{2}$ such that

$$
\begin{aligned}
& \text { (iii) } \int f_{K} \bar{\phi}_{n}=0, n \neq n_{1}, n_{2}, \cdots, n_{K} \text {, and } \\
& \text { (iv) } \int f_{K} \overline{\psi \phi}_{n}=0, n=n_{1}, n_{2}, \cdots, n_{K} \text {. }
\end{aligned}
$$

But (iii) says that there are constants $a_{1}, a_{2}, \cdots, a_{K}$ not all 0 such that $f_{K}=$ ${ }^{a} \phi_{1} \phi_{n_{1}}+a_{2} \phi_{n_{2}}+\cdots+a_{K} \phi_{n_{K}}$, and then we see from (iv) that

$$
\begin{gathered}
a_{1} \int \bar{\psi}\left|\phi_{n_{1}}\right|^{2}+a_{2} \int \bar{\psi} \phi_{n_{2}} \bar{\phi}_{n_{1}}+\cdots+a_{K} \int \bar{\psi} \phi_{n_{K}} \bar{\phi}_{n_{1}}=0 \\
a_{1} \int \bar{\psi} \phi_{n_{1}} \bar{\phi}_{n_{2}}+a_{2} \int \bar{\psi}\left|\phi_{n_{2}}\right|^{2}+\cdots+a_{K} \int \bar{\psi} \phi_{n_{K}} \bar{\phi}_{n_{2}}=0 \\
\vdots \\
a_{1} \int \bar{\psi} \phi_{n_{1}} \bar{\phi}_{n_{K}}+a_{2} \int \bar{\psi} \phi_{n_{2}} \bar{\phi}_{n_{K}}+\cdots+a_{K} \int \bar{\psi}\left|\phi_{n_{K}}\right|^{2}=0 .
\end{gathered}
$$


Suppose now that none of the integrals $\int \bar{\psi} \phi_{n_{i}} \bar{\phi}_{n_{i+1}}, i=1,2, \cdots, K-1$ are 0 . We must show that $\int \bar{\psi} \phi_{n_{K}} \bar{\phi}_{n_{1}}=0$. However, again by the inductive hypothesis, $\int \bar{\psi} \phi_{n_{i}} \bar{\phi}_{n_{j}}=0$ for $i=1,2, \cdots, K-1$ and $j=1,2, \cdots, i$ and also for $i=K$ and $j=2,3, \cdots, K$. By (2) we see that $a_{1}=0$. Then by (3), $a_{2}=0$, etc. Thus, $a_{1}=a_{2}=\cdots=a_{K-1}=0$, and so $a_{K} \neq 0$. Then by $(1), \int \bar{\psi} \phi_{n_{K}} \bar{\phi}_{n_{1}}=0$ as required. By applying the above result to $\delta=\left\{(2 \pi)^{-1 / 2} e^{i n x}\right\}$ we obtain

Theorem 2. There is no finite $\Sigma_{\psi, \delta^{-s e t}}$ if and only if $\psi(z)=z b(z)$ or $\bar{\psi}(z)=$ $z b(z)$ for some function $b$ in $H^{\infty}$ of the unit disk.

Proof. Let us first suppose that $\bar{\psi}(z)=z b(z)$, hence that $\bar{\psi}(x)$ has the Fourier series $\Sigma_{n=1}^{\infty} a_{n} e^{i n x}$ (the proof for the case $\psi=z b$ is identical). Then, for any positive integer $N$, let $\left(n_{1}, n_{2}, \cdots, n_{N}\right)$ be any $N$-tuple of integers, where we can assume that $n_{1} \leq n_{2} \leq \cdots \leq n_{N}$. Then clearly $\exp \left(i n_{N} x\right)$ is orthogonal to $\left\{\theta_{n}(x)\right\}$, and so there is no finite $\Sigma_{\psi, \delta}$-set.

Suppose, on the other hand, that neither $\psi$ nor $\bar{\psi}$ is of the form $z b(z)$, and let the Fourier series of $\bar{\psi}$ be $\Sigma_{j=-\infty}^{\infty} a_{j} e^{i j x}$. If $a_{0} \neq 0$ then clearly any integer is a 1 point $\Sigma_{\psi_{0} \delta}$-set. If $a_{0}=0$ then there exist positive integars $m, n$ such that $a_{-m} a_{n} \neq 0$. In this case we observe that, for the $(m+n)$-tuple $S=\left(n_{1}, n_{2}\right.$, $\left.\cdots, n_{m+n}\right)=(0,-m,-2 m, \cdots,-(n-1) m,-(n-1) m+n,-(n-1) m+2 n, \cdots$, $-(n-1) m+m n)$,

$$
\int \bar{\psi} e^{i n} j^{x} e^{-i n+1}{ }^{x}= \begin{cases}a_{-m} & \text { for } j=1,2, \cdots, n-1, m+n \\ a_{n} & \text { for } j=n, n+1, \cdots, n+m-1 .\end{cases}
$$

That is, for the $(m+n)$-tuple $S$, none of the above integrals are 0 , and thus Theorem 1 assures the existence of a $\Sigma_{\psi, \delta}$-set consisting of at most $m+n$ points.

We are now easily able to prove our Main Theorem. In fact, by Theorem 2, we need only consider the cases where either $\psi$ or $\bar{\psi}$ is of the form $z b(z)$. For convenience we assume the latter case, the proof for the former being identical. Thus $\bar{\psi}(x)$ has the Fourier series $\sum_{n=1}^{\infty} a_{n} e^{i n x}$.

For any integer $M$ we let $S_{M}=\{n: n>M\}$, and we claim that $S_{M}$ is a $\Sigma_{\psi, \delta^{-}}$ set. Indeed, suppose that $f$ is an $L^{2}$ function which is orthogonal to $\left\{\theta_{n}\right\}$, so that

(v) $\int f \bar{\psi} e^{-i n x}=0, n>M$, and

(vi) $\int f e^{-i n x}=0, n \leq M$.

By (v), $f \bar{\psi} \simeq \sum_{n=-\infty}^{M} c_{n} e^{i n x}$ and by (vi), $f \simeq \sum_{n=M+1}^{\infty} b_{n} e^{i n x}$. Thus we have

$$
\sum_{n=M+1}^{\infty} b_{n} e^{i n x} \sum_{n=1}^{\infty} a_{n} e^{i n x} \simeq \sum_{n=-\infty}^{M} c_{n} e^{i n x}
$$

But this implies that $c_{n}=0$ for all $n$, which assures that $f \bar{\psi}=0$ a.e. Since an $H^{\infty}$ function cannot be ${ }^{n} 0$ on a subset of the boundary of positive measure [2, p. 52] 
we see that $f=0$ a.e., and the proof of the Main Theorem is complete.

Remarks. I. We point out that if $Z$ is allowed as a choice for a $\Sigma_{\psi, \delta \text {-set }}$ then the Main Theorem follows immediately from the fact that an $H^{2}$ function cannot be 0 on a subset of the boundary of positive measure. In fact, if $\psi$ is 0 only

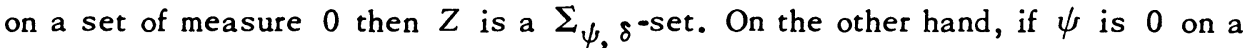
set with measure $\mu$, where $0<\mu<2 \pi$, then the set $S=\{n: n \geq 0\}$ (and of course any translation of $S$ ) will be a $\Sigma_{\psi, \delta}$-set. This is because, if $f$ is orthogonal to $\left\{\theta_{n}\right\}$, then (1) $f \in H^{2}$, and (2) $\bar{f} \bar{\psi}=\overline{z g}$ where $g \in H^{2}$. Thus $g$ is 0 on a set of measure $\mu$, so $g=0$ a.e. Finally, $f$ is 0 on a set of measure $2 \pi-\mu$, so $f=0$ a.e.

II. Many questions remain concerning these ideas. We mention below two which seem particularly interesting and invite the reader to investigate.

(1) It can be shown that if $\psi$ has an absolutely convergent Fourier series then there is a constant $c$ such that $(\psi+c, \delta)$ is a complete pair for the space of continuous functions with period $2 \pi$. Is this condition sufficient for absolute convergence?

(2) What results correspond to the above for spaces of real-valued functions? In this case it appears that these problems require completely different methods.

Appendix. We now prove the "if" half of Theorem 1. The proof is again by induction on $N$.

If $\{n\}$ is a 1 -point $\Sigma_{\psi, \Phi}$-set then it is clear that $\int \psi\left|\phi_{n}\right|^{2} \neq 0$, since if this integral were 0 then $\phi_{n}$ would be orthogonal to $\theta_{m}$ for all $m$, so that $\left\{\theta_{m}\right\}$ would not span $L^{2}$. This completes the case $N=1$.

For the case $N=K$ suppose that for some $m_{0}, 1 \leq m_{0} \leq K$, there is an $m_{0}$

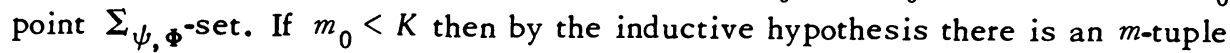
$\left(n_{1}, n_{2}, \cdots, n_{m}\right)$, where $1 \leq m \leq m_{0}$, such that none of the integrals $\int \bar{\psi} \phi_{n_{j}} \bar{\phi}_{n_{j+1}}$, $j=1,2, \cdots, m$, is 0 , and we are done. Thus the only remaining case is $m_{0}=K$, so we suppose that $\left(p_{1}, p_{2}, \cdots, p_{K}\right)$ is a $\Sigma_{\psi, \Phi}$-set. We can assume that for each m-tuple $\left(n_{1}, n_{2}, \cdots, n_{m}\right), 1 \leq m \leq K-1$, at least one of the integrals $\int \bar{\psi} \phi_{n_{j}} \bar{\phi}_{n_{j+1}}$, $j=1,2, \cdots, m$, is 0 . To complete the proof we show that there is some permutation $\left(q_{1}, q_{2}, \cdots, q_{K}\right)$ of $\left(p_{1}, p_{2}, \cdots, p_{K}\right)$ such that none of the integrals $\int \bar{\psi} \phi_{q_{j}} \bar{\phi}_{q_{j+1}}, j=1,2, \cdots, K$, is 0 .

We first let $q_{1}=p_{1}$ and then observe that the completeness of $\left\{\theta_{n}\right\}$ assures the existence of $p_{i}$ for some $i, 1<i \leq K$, such that $\int \bar{\psi} \phi_{q_{1}} \bar{\phi}_{p_{i}} \neq 0$ (otherwise we would have $\phi_{q_{1}}$ orthogonal to $\theta_{n}$ for all $n$ ). We then set $q_{2}=p_{i}$ and observe that by hypothesis $\int \bar{\psi} \phi_{q_{2}} \bar{\phi}_{q_{1}}=0$ (unless $K=2$, in which case $\int \bar{\psi} \phi_{q_{2}} \bar{\phi}_{q_{1}} \neq 0$ by completeness, and the proof is finished). Therefore, again by completeness, for some $j \neq 1, i$ we have $\int \bar{\psi} \phi_{q_{2}} \bar{\phi}_{p_{j}} \neq 0$, and we let $q_{3}=p_{j}$.

Clearly we can continue this process, and we end up with $q_{1}, q_{2}, \cdots, q_{K}$ 
satisfying $\int \bar{\psi} \phi_{q_{i}} \bar{\phi}_{q_{i+1}} \neq 0$ for $i=1,2, \cdots, K-1$. At the final stage we see that $\int \bar{\psi} \phi_{q_{K}} \bar{\phi}_{q_{j}}=0, j=2,3, \cdots, K$ (by considering the $(K-j+1)$-tuple $\left.\left(q_{j}, q_{j+1}, \cdots, q_{K}\right)\right)$, so that, since we cannot have $\phi_{q_{K}}$ orthogonal to $\left\{\theta_{n}\right\}$, $\int \bar{\psi} \phi_{q_{K}} \bar{\phi}_{q_{1}} \neq 0$. Thus $\left(q_{1}, q_{2}, \cdots, q_{K}\right)$ satisfies the required property, and the proof is complete.

\section{REFERENCES}

1. J. S. Byrnes and D. J. Newman, Completeness preserving multipliers, Proc. Amer. Math. Soc. 21 (1969), 445-450. MR $39 \# 1893$.

2. Kenneth Hoffman, Banach spaces of analytic functions, Prentice-Hall Series in Modern Analysis, Prentice-Hall, Englewood Cliffs, N. J., 1962. MR 24 \#A2844.

DEPARTMENT OF MATHEMATICS, UNIVERSITY OF MASSACHUSETTS, BOSTON, MASSACHUSETTS 02116

NAVAL UNDERWATER SYSTEMS CENTER, NEW LONDON, CONNECTICUT 06320 